\title{
Nutritional status of patients treated with radiotherapy as determined by subjective global assessment
}

\author{
Woong Sub Koom, MD', Seung Do Ahn, MD², Si Yeol Song, MD², Chang Geol Lee, MD²,

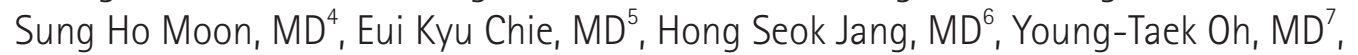 \\ Ho Sun Lee, $\mathrm{RD}^{8}$, Ki Chang Keum, MD ${ }^{1}$ \\ Department of Radiation Oncology, ${ }^{1}$ Yonsei University College of Medicine, Seoul, ${ }^{2}$ Asan Medical Center, Seoul, \\ ${ }^{3}$ Gangnam Severance Hospital, Yonsei University College of Medicine, Seoul, ${ }^{4}$ Research Institute and Hospital, \\ National Cancer Center, Goyang, ${ }^{5}$ Seoul National University College of Medicine, Seoul, ${ }^{6}$ Seoul St. Mary's Hospital, The Catholic \\ University of Korea College of Medicine, Seoul, and ${ }^{7}$ Ajou University School of Medicine, Suwon; ${ }^{8}$ Department of Nutrition \\ and Dietetics, Severance Hospital, Yonsei University Health System, Seoul, Korea
}

Purpose: The purpose of this prospective multi-institutional study was to evaluate the nutritional status of patients undergoing radiotherapy (RT) for treatment of head and neck, lung, or gastrointestinal cancer.

Materials and Methods: A total of 1,000 patients were enrolled in this study at seven different hospitals in Seoul, Korea between October 2009 and May 2010. The nutritional status of patients after receiving 3 weeks of RT was evaluated using subjective global assessment (SGA). The nutritional status of each patient was rated as well nourished (A), moderately malnourished (B), or severely malnourished (C).

Results: The mean age of patients in this study was $59.4 \pm 11.9$ years, and the male to female ratio was $7: 3$. According to the SGA results, $60.8 \%, 34.5 \%$, and $4.7 \%$ of patients were classified as $A, B$, or $C$, respectively. The following criteria were significantly associated with malnutrition (SGA B or $C_{i} p<0.001$ ): loss of subcutaneous fat or muscle wasting (odds ratio [OR], 11.473); increased metabolic demand/stress (OR, 8.688); ankle, sacral edema, or ascites (OR, 3.234); and weight loss $\geq 5 \%(O R, 2.299)$.

Conclusion: SGA was applied successfully to assess the nutritional status of most patients. The prevalence of malnutrition in a radiation oncology department was 39.2\%. The results of this study serve as a basis for implementation of nutrition intervention to patients being treated at radiation oncology departments.

Keywords: Malnutrition, Nutrition assessment, Subjective global assessment, Radiotherapy

\section{Introduction}

Malnutrition is a potentially serious condition often comorbid with cancer and its treatment. The incidence of malnutrition in cancer patients has been reported to range from about 10\% to $80 \%$ and malnutrition itself was one of the reasons for death in up to $20 \%$ of cancer patients [1-3]. Malnutrition contributes to an increased risk of toxicity, infection, and healthcare costs, as well as decreased treatment response, compliance, quality of life, and ultimately patient survival [4-6]. Given the importance of nutritional status, screening patients at risk for malnutrition and providing a nutrition support program is of great concern

Received 23 August 2012, Revised 17 September 2012, Accepted 21 September 2012.

Correspondence: Ki Chang Keum, MD, Department of Radiation Oncology, Yonsei University College of Medicine, 50 Yonsei-ro, Seodaemun-gu, Seoul 120-752, Korea. Tel: +82-2-2228-8112, Fax: +82-2-312-9033, E-mail: kckeum@yuhs.ac

(c) This is an Open Access article distributed under the terms of the Creative Commons Attribution Non-Commercial License (http://creativecommons.org/ licenses/by-nc/3.0/) which permits unrestricted non-commercial use, distribution, and reproduction in any medium, provided the original work is properly cited. www.e-roj.org 
for patients with cancer [7]. Subjective global assessment (SGA) is a validated clinical tool for assessing nutritional status and is based on the features of a medical history and physical examination [8]. First described more than two decades ago, SGA is still being successfully used as a standardized method of assessing nutritional status in various patient populations, including those with cancer [7-12]. Furthermore, nutritional status using SGA was associated with prognosis and quality of life in cancer patients [13-15].

The acute reaction of the aerodigestive tract as a result of undergoing radiotherapy (RT) is associated with diverse gastrointestinal symptoms and decreased food intake, resulting in deterioration of the patient's nutritional status [16]. Several prospective, randomized trials have reported the effectiveness of early nutritional intervention on the quality of life in cancer patients undergoing RT [17-20]. Despite the frequent occurrence of nutritionally related side effects, a nutritional assessment of patients with cancer receiving $\mathrm{RT}$ is not routine practice in the clinical setting. There has been no study as of yet investigating the prevalence of malnutrition during

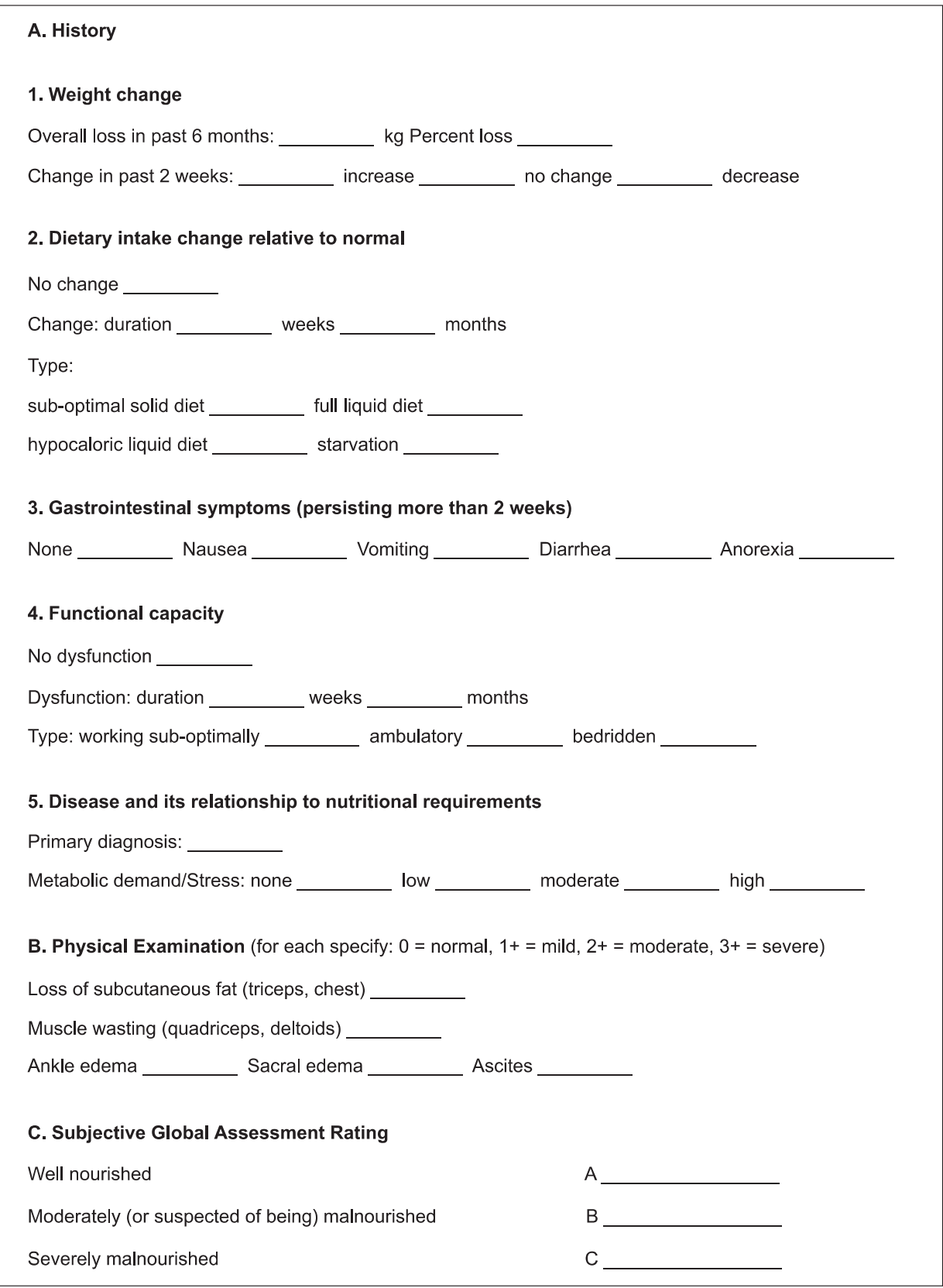

Fig. 1. Feature of subjective global assessment by Detsky et al. [8]. 
Table 1. Patients and nutritional characteristics of all subjects

\begin{tabular}{|c|c|c|}
\hline \multicolumn{2}{|l|}{ Characteristic } & \multirow{2}{*}{$\begin{array}{c}\text { Value } \\
59.4 \pm 11.9\end{array}$} \\
\hline Age (yr) & & \\
\hline \multirow[t]{2}{*}{ Sex } & Male & $703(70.3)$ \\
\hline & Female & $297(29.7)$ \\
\hline \multirow[t]{3}{*}{ Tumor site } & Head and neck & $286(28.6)$ \\
\hline & $\begin{array}{l}\text { Gastrointesti- } \\
\text { nal tract }\end{array}$ & $444(44.4)$ \\
\hline & Lung & $270(27.0)$ \\
\hline \multirow[t]{2}{*}{ Chemotherapy } & No & $528(52.8)$ \\
\hline & Yes & $472(47.2)$ \\
\hline Weight loss in past 6 mo (kg) & & $2.1 \pm 3.9$ \\
\hline Percent weight loss in past 6 mo (\%) & & $3.9 \pm 4.8$ \\
\hline \multirow[t]{4}{*}{ Weight change in previous 2 wk } & Increase & $110(11.0)$ \\
\hline & No change & $590(59.0)$ \\
\hline & Decrease & $275(27.5)$ \\
\hline & Unknown & $25(2.5)$ \\
\hline \multirow[t]{2}{*}{ Dietary intake change } & No & $667(66.7)$ \\
\hline & Yes & $331(33.1)$ \\
\hline Duration of diet change (wk) & & $3.8 \pm 7.3$ \\
\hline \multirow[t]{4}{*}{ Type of diet change } & $\begin{array}{l}\text { Suboptimal } \\
\text { solid diet }\end{array}$ & $174(17.4)$ \\
\hline & Liquid diet & $66(6.6)$ \\
\hline & Starvation & $6(0.6)$ \\
\hline & Unknown & $85(8.5)$ \\
\hline \multirow[t]{2}{*}{ Nausea } & No & $701(70.1)$ \\
\hline & Yes & $299(29.9)$ \\
\hline \multirow[t]{2}{*}{ Vomiting } & No & 909 (90.9) \\
\hline & Yes & $91(9.1)$ \\
\hline \multirow[t]{2}{*}{ Diarrhea } & No & $912(91.2)$ \\
\hline & Yes & $88(8.8)$ \\
\hline \multirow[t]{2}{*}{ Anorexia } & No & $494(49.4)$ \\
\hline & Yes & $506(50.6)$ \\
\hline \multirow[t]{2}{*}{ Functional capacity } & No & $619(61.9)$ \\
\hline & Yes & $380(38.0)$ \\
\hline Duration of dysfunction (wk) & & $9.2 \pm 26.3$ \\
\hline \multirow{4}{*}{ Type of dysfunction } & Suboptimal & $222(22.2)$ \\
\hline & Ambulatory & $134(13.4)$ \\
\hline & Bedridden & $14(1.4)$ \\
\hline & Unknown & $10(1.0)$ \\
\hline \multirow[t]{5}{*}{ Metabolic demand/stress } & No & $123(12.3)$ \\
\hline & Low & $661(66.1)$ \\
\hline & Moderate & $174(17.4)$ \\
\hline & High & $32(3.2)$ \\
\hline & Unknown & $10(1.0)$ \\
\hline \multirow[t]{4}{*}{ Loss of subcutaneous fat } & Normal & $631(63.1)$ \\
\hline & Mild & $254(25.4)$ \\
\hline & Moderate & $104(10.4)$ \\
\hline & Severe & $11(1.1)$ \\
\hline
\end{tabular}

Table 1. Continued

\begin{tabular}{llc}
\hline \multicolumn{2}{c}{ Characteristic } & Value \\
\hline Muscle wasting & Normal & $675(67.5)$ \\
& Mild & $221(22.1)$ \\
& Moderate & $91(9.1)$ \\
& Severe & $12(1.2)$ \\
Unknown & $1(0.1)$ \\
Normal & $901(90.1)$ \\
Ankle edema & Mild & $62(6.2)$ \\
& Moderate & $33(3.3)$ \\
& Severe & $4(0.4)$ \\
& Normal & $915(91.5)$ \\
Sacral edema & Mild & $48(4.8)$ \\
& Moderate & $33(3.3)$ \\
& Severe & $4(0.4)$ \\
Ascites & Normal & $902(90.2)$ \\
& Mild & $44(4.4)$ \\
& Moderate & $34(3.4)$ \\
& Severe & $4(0.4)$ \\
& Unknown & $16(1.6)$ \\
Subjective global assessment & Well nourished & $608(60.8)$ \\
& Moderately & $345(34.5)$ \\
& malnourished & \\
& Severely & $47(4.7)$ \\
& malnourished & \\
\hline
\end{tabular}

Values are presented as mean \pm standard deviation or number (\%).

radiotherapy in Korea. This prospective multi-institutional study was performed to evaluate the nutritional status using SGA in ambulatory patients undergoing RT to the head, neck, thorax, abdomen, and pelvic area.

\section{Materials and Methods}

\section{Participants}

This prospective study was designed to investigate the nutritional status of patients with cancer receiving RT Between October 2009 and May 2010, consecutive 1,000 patients were enrolled in seven different hospitals in Seoul, Korea. Eligibility criteria included: 1) having primary cancer of the head and neck, lung, or gastrointestinal tract; 2) receiving RT over a period of 3 weeks to the primary tumor site, regardless of RT intent (primary, adjuvant to surgery, combined with chemotherapy, or palliation); and 3) willing and able to give written informed consent. We concerned the acute radiation response of the aerodigestive tract. So, the patients treated with RT over 3 weeks to the head and neck, lung, or gastrointestinal tract were enrolled. Patients who were 
Table 2. Patient and nutritional characteristics of subjects according to subjective global assessment category

\begin{tabular}{|c|c|c|c|c|}
\hline Characteristic & Status & Well nourished $(A)$ & Malnourished (B and C) & p-value \\
\hline \multirow[t]{3}{*}{ Age (yr) } & & & & 0.116 \\
\hline & $<60$ & $293(48.2)$ & $169(43.1)$ & \\
\hline & $\geq 60$ & 315 (51.8) & $223(56.9)$ & \\
\hline \multirow[t]{3}{*}{ Sex } & & & & 0.232 \\
\hline & Male & $419(68.9)$ & $284(72.4)$ & \\
\hline & Female & $189(31.1)$ & $108(27.6)$ & \\
\hline \multirow[t]{4}{*}{ Tumor site } & & & & 0.799 \\
\hline & Head and neck & $172(28.3)$ & $114(29.1)$ & \\
\hline & Gastrointestinal tract & $275(45.2)$ & $169(43.1)$ & \\
\hline & Lung & $161(26.5)$ & $109(27.8)$ & \\
\hline \multirow[t]{3}{*}{ Chemotherapy } & & & & 0.006 \\
\hline & No & $342(56.3)$ & $186(47.4)$ & \\
\hline & Yes & 266 (43.8) & $206(52.6)$ & \\
\hline \multirow[t]{3}{*}{ Percent weight loss in past 6 mo (\%) } & & & & $<0.001$ \\
\hline & $<5$ & $496(81.6)$ & $167(42.6)$ & \\
\hline & $\geq 5$ & $108(17.8)$ & $223(56.9)$ & \\
\hline \multirow[t]{3}{*}{ Weight change in previous $2 \mathrm{wk}$} & & & & $<0.001$ \\
\hline & Increase or no change & $496(81.6)$ & $204(52.0)$ & \\
\hline & Decrease & $100(16.4)$ & $175(44.6)$ & \\
\hline \multirow[t]{3}{*}{ Dietary intake change } & & & & $<0.001$ \\
\hline & No & $478(78.6)$ & $189(48.2)$ & \\
\hline & Yes & $130(21.4)$ & 201 (51.3) & \\
\hline \multirow[t]{3}{*}{ Nausea } & & & & $<0.001$ \\
\hline & No & $464(76.3)$ & $237(60.5)$ & \\
\hline & Yes & $144(23.7)$ & 155 (39.5) & \\
\hline \multirow[t]{3}{*}{ Vomiting } & & & & $<0.001$ \\
\hline & No & $588(96.7)$ & 321 (81.9) & \\
\hline & Yes & $20(3.3)$ & $71(18.1)$ & \\
\hline \multirow[t]{3}{*}{ Diarrhea } & & & & 0.016 \\
\hline & No & 565 (92.9) & 347 (88.5) & \\
\hline & Yes & $43(7.1)$ & $45(11.5)$ & \\
\hline \multirow[t]{3}{*}{ Anorexia } & & & & $<0.001$ \\
\hline & No & $373(61.3)$ & $121(30.9)$ & \\
\hline & Yes & $235(38.7)$ & $271(69.1)$ & \\
\hline \multirow[t]{3}{*}{ Functional capacity } & & & & $<0.001$ \\
\hline & No & $423(69.6)$ & $196(50.0)$ & \\
\hline & Yes & $185(30.4)$ & $195(49.7)$ & \\
\hline \multirow[t]{3}{*}{ Metabolic demand/stress } & & & & $<0.001$ \\
\hline & No or low & 570 (93.8) & $214(54.6)$ & \\
\hline & Moderate or high & $36(5.9)$ & $170(43.4)$ & \\
\hline \multirow[t]{3}{*}{ Loss of subcutaneous fat } & & & & $<0.001$ \\
\hline & No & 528 (86.8) & $103(26.3)$ & \\
\hline & Yes & $80(13.2)$ & $289(73.7)$ & \\
\hline \multirow[t]{3}{*}{ Muscle wasting } & & & & $<0.001$ \\
\hline & No & $525(86.3)$ & $150(38.3)$ & \\
\hline & Yes & $83(13.7)$ & $241(61.5)$ & \\
\hline \multirow[t]{3}{*}{ Ankle edema } & & & & $<0.001$ \\
\hline & No & $576(94.7)$ & 325 (82.9) & \\
\hline & Yes & $32(5.3)$ & $67(17.1)$ & \\
\hline
\end{tabular}


Table 2. Continued

\begin{tabular}{|c|c|c|c|c|}
\hline Characteristic & Status & Well nourished (A) & Malnourished (B and $C$ ) & $p$-value \\
\hline \multirow[t]{3}{*}{ Sacral edema } & & & & $<0.001$ \\
\hline & No & $578(95.1)$ & 337 (86.0) & \\
\hline & Yes & $30(4.9)$ & 55 (14.0) & \\
\hline \multirow[t]{3}{*}{ Ascites } & & & & $<0.001$ \\
\hline & No & $563(92.6)$ & $339(86.5)$ & \\
\hline & Yes & $30(4.9)$ & $52(13.3)$ & \\
\hline
\end{tabular}

Values are presented as number (\%).

obviously moribund, not willing to participate, or not able to respond during interview were excluded. The ethics committee of each study hospital approved the study protocol.

\section{Nutritional assessment and data collection}

The nutritional status of each patient was assessed 3 weeks after the initiation of RT, and was determined using SGA tool [8]. The 3-week period between RT commencement and nutritional assessment was chosen because RT-induced acute toxicity, such as mucositis, develops during this period. The SGA tool is based on a medical history (weight loss; dietary intake change; gastrointestinal symptoms including nausea, vomiting, diarrhea and anorexia; and changes in functional capacity) and physical examination (loss of subcutaneous fat; muscle wasting; ankle edema, sacral edema, and ascites). Each patient was classified as either well nourished (SGA A), moderately or suspected of being malnourished (SGA B), or severely malnourished (SGA C). This classification was assigned on the basis of subjective weighting. The details of SGA assessment was described by Detsky et al. [8] (Fig. 1). For the purpose of this analysis, malnutrition was defined as either SGA B or SGA C. A single trained rater (physician or dietitian) in each hospital assessed nutritional status. In order to minimize inter-rater variation, all raters were educated by the principle dietician of the coordinating study center. In addition to recording nutritional information, the following information was collected from the medical record within 4 weeks before or during RT: age, sex, diagnosis, and chemotherapy.

\section{Statistical analysis}

All continuous variables and categorical variables were transformed into two categorical variables. Univariate analysis was conducted by performing chi-square tests to compare the association between SGA criteria and malnutrition. To identify independent risk factors for malnutrition, binary logistic regression analysis was performed. Significance was defined as $p<0.05$. Data were analyzed using SPSS ver. 18.0 (SPSS Inc. Chicago, IL, USA).

\section{Results}

\section{Characteristics and nutritional status of participants}

Baseline characteristics of the participants are shown in Table 1. The mean age of enrolled subjects was $59.4 \pm 11.9$ years. Of the total 1,000 patients, 703 (70.3\%) were men and 293 (29.3\%) were women. Gastrointestinal cancer, including esophageal cancer, was the most the common parameter and was present in 444 of the patients. Head and neck cancer and lung cancer affected 286 patients and 270 patients, respectively. Chemotherapy was performed either during RT or within the 4 weeks before RT in 472 patients. According to SGA, 608 (60.8\%), 345 (34.5\%), and 47 (4.7\%) patients were SGA A, SGA B, and $S G A C$, respectively.

\section{Impact of SGA parameters and clinical factors on malnutrition}

Table 2 shows the results of univariate analysis of clinical factors and SGA parameters for each SGA classification. For this analysis, SGA B and C were grouped together in the malnourished category. Additionally, all continuous variables were transformed into categorical variables. Except for tumor site, all categorical variables were re-coded into two groups. There was no significant association between malnutrition (SGA $B$ and $C)$ and age $(p=0.116)$, sex $(p=0.232)$, or tumor site ( $p=0.799$ ). Chemotherapy in addition to all SGA parameters were significantly associated with malnutrition (SGA B and C). In a multivariate analysis, similar SGA parameters were coded as one (Table 3). Loss of subcutaneous fat or muscle wasting strongly was associated with the development of malnutrition (odds ratio [OR], 11.473; $p<0.001$ ). Metabolic demand/stress was the next most strongly contributing factor $(O R, 8.688 ; p$ $<0.001)$. Patients with ankle, sacral edema, or ascites upon 
Table 3. Independent risk factors for malnourishment (subjective global assessment $\mathrm{B}$ and $\mathrm{C}$ )

\begin{tabular}{lcr}
\hline & OR $(95 \% \mathrm{Cl})$ & p-value \\
\hline Chemotherapy & $1.180(0.821-1.697)$ & 0.370 \\
Percent weight loss in past & $2.299(1.567-3.373)$ & $<0.001$ \\
6 mo & & \\
Dietary intake change & $1.407(0.911-2.172)$ & 0.124 \\
Nausea/vomiting & $1.080(0.703-1.661)$ & 0.724 \\
Diarrhea & $1.412(0.723-2.756)$ & 0.312 \\
Anorexia & $2.018(1.356-3.004)$ & 0.001 \\
Functional capacity & $1.105(0.736-1.658)$ & 0.632 \\
Metabolic demand/stress & $8.688(5.319-14.192)$ & $<0.001$ \\
Loss of subcutaneous fat or & $11.473(7.588-17.347)$ & $<0.001$ \\
$\quad$ muscle wasting & & \\
Ankle, sacral edema or & $3.234(1.716-6.094)$ & $<0.001$ \\
ascites & & \\
\hline
\end{tabular}

$\mathrm{OR}$, odds ratio; $\mathrm{Cl}$, confidence interval.

physical examination were also at a higher risk of being malnourished (OR, 3.234; $p<0.001)$. Additionally, patients with $\geq 5 \%$ weight loss in the previous 6 months were also more likely than other patients to be malnourished (OR, 2.299; $p<0.001)$.

\section{Discussion and Conclusion}

Three weeks after initiation of RT, we used SGA to evaluate the nutritional status of ambulatory patients with cancer whose site of treatment varied. SGA was applied successfully to assess the nutritional status of most patients. The prevalence of patients who were malnourished or at risk of becoming malnourished while being treated at one of the seven hospitals in the study population was high at 39.2\%.

Malnutrition is common in cancer patients, and has been reported to occur in up to $80 \%$ of patients treated with RT. Furthermore, malnutrition is associated with increased morbidity and mortality $[2,6]$. It has been suggested that malnutrition increases the risk of infections caused by immune dysfunction, as well as decreasing quality of life and survival [4]. Moreover, malnutrition adversely affects patients' response to cancer treatment and increases the incidence of treatmentrelated toxicities. Oncologic treatment exacerbates acute and chronic symptoms due to issues with poor food intake and poor nutritional status [21]. Most of the RT-related toxicities are closely associated with nutritional problems [16]. Given the deteriorating side effect of RT, several prospective, randomized trials have been conducted to evaluate the effectiveness of nutritional intervention on the quality of life as well as nutritional status in patients with cancer undergoing RT [17-20,22,23]. Consistently, the nutritional intervention positively influenced weight, nutritional status, and quality of life compared to usual care in patients receiving RT to the gastrointestinal or head and neck area $[17,19,20,22]$. Therefore, early identification of malnutrition in cancer patients and nutritional intervention may increase tolerance to cancer treatment and improve quality of life and prognosis $[3,4,24,25]$.

Many nutrition screening tools have been proposed that combine multiple components, including dietary and medical history, weight loss, biochemical indicators of body protein, and anthropometry [26]. SGA for determining nutritional status was developed by Detsky et al. [8] in 1987. This assessment tool is simple, non-invasive, and inexpensive, consisting of a medical history (weight change, dietary intake change, gastrointestinal symptoms, and changes in functional capacity) and a physical examination (loss of subcutaneous fat, muscle wasting, ankle edema, sacral edema, and ascites). SGA categorizes patients into three nutritional status groups: well nourished (SGA A), moderately malnourished (SGA B), and severely malnourished ( $S G A C$ ).

$\mathrm{SGA}$ is a reliable tool that that enables correlation of a number of objective nutritional assessment indicators, such as morbidity, mortality, and quality of life ( $\mathrm{O} o \mathrm{~L})$ measures, and has been used universally in diverse clinical settings, various regions, and multicenter studies [7,9-12,27,28]. A limitation of using SGA is that it only classifies subjects into three general groups, and it does not reflect subtle changes in nutritional status. Ottery [7] developed a patient-generated SGA tool using a scoring system for patients with cancer that allowed data to be expressed as a continuous measurement. Using this tool, investigators can detect subtle changes of nutritional status. For more than two decades, however, the original SGA has been used to assess patient malnutrition in several clinical settings and is the standard method [27]. In this study, we have successfully conducted nutritional surveys using SGA in multiple institutions, using rater education to support optimal consistency. According to SGA, the prevalence of malnutrition in this study population was $39.2 \%$. This finding is similar to the $35 \%$ of patients who were malnourished in the Australian Radiation Oncology Facilities as determined by patientgenerated SGA [28]. We demonstrated the malnutrition in large number of ambulatory patients undergoing RT. This is first step to raise concern about nutrition assessment at 
radiation oncology departments.

The group of patients enrolled this study is very heterogeneous and includes various levels of tumor burden and RT protocol across multiple institutions. In addition, RT parameters such as dose and volume were variable. RT-related toxicities associated with nutritional problems were not be evaluated. This study did not investigate inter-rater reliability or reproducibility. To minimize observer bias, SGA assessment was performed after a training period that took place over a few days. A well-trained observer can distinguish between malnourished (SGA categories B and C) and normal patients, although they had more difficulty discriminating between moderate and severe malnutrition [29]. This study applied the SGA only at one time point and is observational because no nutritional intervention was performed. Nevertheless, our study analyzed a large population of patients with cancer receiving $\mathrm{RT}$ and adds the clinically useful information that malnutrition is an important problem in cancer patients undergoing RT. Further studies are needed to evaluate the effectiveness of nutritional intervention at several time points in a homogenous group of patients to determine the benefit of nutritional support on clinical outcomes in cancer patients treated with RT. In conclusion, this study identified the prevalence of malnutrition using the SGA tool in patients treated with RT to the head and neck, thorax, abdomen and pelvic area. Thirty-nine point two percent of patients were moderately or suspected of being malnourished or severely malnourished. The results of this study serve as a basis for implementation of nutrition intervention to patients being treated at radiation oncology departments.

\section{Conflict of Interest}

No potential conflict of interest relevant to this article was reported.

\section{Acknowledgments}

This study was supported by an Educational Grant from Abbott Korea Ltd. (Seoul, Korea).

\section{References}

1. Laviano A, Meguid MM. Nutritional issues in cancer management. Nutrition 1996;12:358-71.

2. Ollenschlager $G$, Viell B, Thomas $W$, Konkol K, Burger B. Tumor anorexia: causes, assessment, treatment. Recent Results
Cancer Res 1991;121:249-59.

3. Ottery FD. Cancer cachexia: prevention, early diagnosis, and management. Cancer Pract 1994;2:123-31.

4. Nitenberg G, Raynard B. Nutritional support of the cancer patient: issues and dilemmas. Crit Rev Oncol Hematol 2000;34:137-68.

5. Ottery FD. Definition of standardized nutritional assessment and interventional pathways in oncology. Nutrition 1996; 12:S15-9.

6. Rivadeneira DE, Evoy D, Fahey TJ 3rd, Lieberman MD, Daly JM. Nutritional support of the cancer patient. CA Cancer J Clin 1998;48:69-80.

7. Ottery FD. Rethinking nutritional support of the cancer patient: the new field of nutritional oncology. Semin Oncol 1994;21:770-8.

8. Detsky AS, McLaughlin JR, Baker JP, et al. What is subjective global assessment of nutritional status? JPEN J Parenter Enteral Nutr 1987;11:8-13.

9. Bauer J, Capra S, Ferguson M. Use of the scored PatientGenerated Subjective Global Assessment (PG-SGA) as a nutrition assessment tool in patients with cancer. Eur J Clin Nutr 2002;56:779-85.

10. Duerksen DR, Yeo TA, Siemens JL, O'Connor MP. The validity and reproducibility of clinical assessment of nutritional status in the elderly. Nutrition 2000;16:740-4.

11. Ferguson ML, Bauer J, Gallagher B, Capra S, Christie DR, Mason BR. Validation of a malnutrition screening tool for patients receiving radiotherapy. Australas Radiol 1999;43:3257.

12. Thoresen L, Fjeldstad I, Krogstad K, Kaasa S, Falkmer UG. Nutritional status of patients with advanced cancer: the value of using the subjective global assessment of nutritional status as a screening tool. Palliat Med 2002;16:33-42.

13. Gupta D, Lammersfeld CA, Vashi PG, Burrows J, Lis CG, Grutsch JF. Prognostic significance of Subjective Global Assessment (SGA) in advanced colorectal cancer. Eur J Clin Nutr 2005;59:35-40.

14. Gupta D, Lammersfeld CA, Vashi PG, Dahlk SL, Lis CG. Can subjective global assessment of nutritional status predict survival in ovarian cancer? J Ovarian Res 2008;1:5.

15. Gupta D, Lis CG, Granick J, Grutsch JF, Vashi PG, Lammersfeld CA. Malnutrition was associated with poor quality of life in colorectal cancer: a retrospective analysis. J Clin Epidemiol 2006:59:704-9.

16. Capra S, Ferguson M, Ried K. Cancer: impact of nutrition intervention outcome - nutrition issues for patients. Nutrition 2001;17:769-72.

17. Isenring EA, Capra $S$, Bauer JD. Nutrition intervention is beneficial in oncology outpatients receiving radiotherapy to the gastrointestinal or head and neck area. Br J Cancer 
2004;91:447-52.

18. Ravasco P, Monteiro-Grillo I, Camilo ME. Does nutrition influence quality of life in cancer patients undergoing radiotherapy? Radiother Oncol 2003;67:213-20.

19. Ravasco P, Monteiro-Grillo I, Marques Vidal P, Camilo ME. Impact of nutrition on outcome: a prospective randomized controlled trial in patients with head and neck cancer undergoing radiotherapy. Head Neck 2005;27:659-68.

20. Ravasco P, Monteiro-Grillo I, Vidal PM, Camilo ME. Dietary counseling improves patient outcomes: a prospective, randomized, controlled trial in colorectal cancer patients undergoing radiotherapy. J Clin Oncol 2005;23:1431-8.

21. Van Cutsem $E$, Arends J. The causes and consequences of cancer-associated malnutrition. Eur J Oncol Nurs 2005;9 Suppl 2:S51-63.

22. Isenring EA, Bauer JD, Capra S. Nutrition support using the American Dietetic Association medical nutrition therapy protocol for radiation oncology patients improves dietary intake compared with standard practice. J Am Diet Assoc 2007;107:404-12.
23. Unsal D, Mentes B, Akmansu M, Uner A, Oguz M, Pak $Y$. Evaluation of nutritional status in cancer patients receiving radiotherapy: a prospective study. Am J Clin Oncol 2006;29:183-8.

24. Marin Caro MM, Laviano A, Pichard C. Nutritional intervention and quality of life in adult oncology patients. Clin Nutr 2007;26:289-301.

25. Ottery FD. Supportive nutrition to prevent cachexia and improve quality of life. Semin Oncol 1995;22:98-111.

26. Makhija S, Baker J. The subjective global assessment: a review of its use in clinical practice. Nutr Clin Pract 2008;23:405-9.

27. Barbosa-Silva MC, Barros AJ. Indications and limitations of the use of subjective global assessment in clinical practice: an update. Curr Opin Clin Nutr Metab Care 2006;9:263-9.

28. Isenring E, Bauer J, Capra S. The scored Patient-generated Subjective Global Assessment (PG-SGA) and its association with quality of life in ambulatory patients receiving radiotherapy. Eur J Clin Nutr 2003;57:305-9.

29. Duerksen DR. Teaching medical students the subjective global assessment. Nutrition 2002;18:313-5. 